\section{METROPOLIS}

Texte und Studien zu Zentren der Kultur in der europäischen Neuzeit

\section{Herausgegeben von}

Sandra Richter, Johann Anselm Steiger und Axel E. Walter

\section{HAMBURG}

Eine Metropolregion zwischen Früher Neuzeit und Aufklärung

Herausgegeben von

Johann Anselm Steiger und Sandra Richter

\title{
Akademie Verlag
}




\section{„In die äusserste Welt Oerther“}

Die Hamburger Kaufmannschaft und ihre frühneuzeitlichen

Handelsbeziehungen

\section{Zusammenfassung}

Hamburg unterhielt in der Frühen Neuzeit intensive Handelsbeziehungen zu einer Vielzahl europäischer, amerikanischer, afrikanischer und möglicherweise auch asiatischer Häfen. Die Bedeutung der Stadt und ihrer Kaufleute wurde bislang jedoch kaum im globalen Zusammenhang untersucht. Die existierenden Arbeiten beschränken sich auf Teilaspekte und betrachten den hamburgischen Handel vor einem ,nationalen' Hintergrund. Hamburg verfolgte in der Frühen Neuzeit jedoch eine weitgehend unabhängige Außen- und Wirtschaftspolitik, und die hamburgischen Kaufleute, die im Ausland zum Teil einflussreiche Positionen besetzten, wurden dort als eigenständige Nation betrachtet. Der vorliegende Beitrag plädiert dafür, die Perspektive der lokalen Geschichtsschreibung zu erweitern und die Stadt sowie ihre soziale, wirtschaftliche und kulturelle Entwicklung im globalen Kontext zu untersuchen.

In der 1668 erschienenen Schrift Hamburgs Hoheit des deutsch-dänischen Schriftstellers Conrad von Hövelen heißt es:

Mit allerhand Kaufardei- und auch Orlog-Schiffen ist Hamburg wol und reichlich gefullet, so teils in die äusserste Welt Oerther in Ost- und West-Indien, America, Asia und Africa, samt Europa aus- und mit gutem Gewinne wider ein- und anheime fligen. ${ }^{1}$

Der Dichter und Zeitungsredakteur Georg Greflinger berichtete sechs Jahre später vom

continuirlichen Auf- und Abgang der unzählichen grossen und kleinen Schiffe, dere rosse meistens aus Spanien, auch aus der Straten, aus Portugal, aus Franckreich, Engeland, Schott- und Irrland, aus Dennemarck, Schweden, Norwegen, Holl- und See-Land, und aus der Ost-See, auch von Archangel und Grönland, ja Guinea und America kommen, und eines jeden Landes Früchte und Wahren reichlich einbringen: Als aus Spanien und Portugal Weine, Saltz und andere herrliche Gewächse. Aus der Straten seidene und andere Wahren. Aus Frankreich Weine, Castanien, Galanterien und anders. Aus Engeland und Schottland allerley Lacken und Stoffe zu Kleidungen, seiden und wollen Strümpffe, Zinn, Bley und gut Bier, etc. Aus Dennemarck und Norwegen gesaltzen len Strümpffe, Zinn, Bley und gut Bier, etc. Aus Dennemarck und Norwegen gesaltzen
Fleisch, Fische, Holtz, Häute, und anders. Aus Schweden Kupffer, Eisen, Geschütze, und schöne Steine. Aus Holl- und Seeland theure Stück-Güter, Hering, Käse, Butter und anders. Aus Grünland Walfische zum Tran und Bahren (sind Fischbeine). Aus Archange köstliche Belzereyen, Juchten, Lächse, Caviar, und andere Wahren. Aus Gvinea Gold,

1 Conrad von Hövelen: Der Uhr-alten Deutschen Grossen und des H: Röm: Reichs- Freien AnSee- und Handel-Stadt Hamburg Alt-vorige und noch Iz Zu-Nämende Hoheit. Lübeck 1668 , $75 \mathrm{f}$ 
Helffenbein, Meer-Kazen, Papagoyen, Affen, Pavianen und anders. Aus America Toback, Zucker, Catun, Campez-Holtz, Indigo und andere köstliche Wahren. ${ }^{2}$

In der Tat hatte sich Hamburg seit dem ausgehenden 16. Jahrhundert einen wichtigen Platz in der Weltwirtschaft erobert. Bereits 1558 war die Hamburger Börse gegründet worden. Mit der Wechselordnung von 1605 wurde der rechtliche Rahmen für den reibungslosen Ablauf des bargeldlosen Zahlungsverkehrs geschaffen Gleichzeitig entwickelte sich, wenn auch zögerlich, ein Seeversicherungsmarkt. 1619 erfolgte die Gründung einer öffentlichen Bank. Eine große Zahl von Maklern und Notaren bot den Kaufleuten ihre Dienste in den verschiedensten Sprachen an. 1580 war eine Botenordnung in Hamburg eingeführt worden, die die Grundlage für einen zuverlässigen und regelmäßigen Postverkehr legte. Seit 1607 gab es genaue Abgangs- und Ankunftszeiten der Briefe für alle Poststrecken. Die Kaufleute konnten nun ihre Geschäftspartner in ganz Europa innerhalb eines relativ genau kalkulierbaren Zeitraums über wichtige Ereignisse und Entscheidungen informie ren. Als internationales Nachrichtenzentrum, in dem viele diplomatische Vertrete residierten, wurde Hamburg zudem zu einem der führenden Zeitungsdruckorte Europas. Die Berichterstattung reichte weit über die europäischen Grenzen hinaus und umfasste beispielsweise auch die in Brasilien ausgetragenen militärischen Konflikte zwischen Holland und Portugal.

Den Dreißigjährigen Krieg überstand die Stadt nicht nur unversehrt, sondern konnte durch den Rüstungshandel und die Finanzierung militärischer Unternehmungen sogar wirtschaftlich von ihm profitieren. ${ }^{4}$ Nachdem sich die Hamburger Bevölkerung bereits zwischen 1550 und 1600 fast verdoppelt hatte und die Stadt um die Jahrhundertwende rund 40.000 Einwohner zählte, wuchs sie bis zum Ende des 17. Jahrhunderts auf knapp 80.000 Personen an. ${ }^{5}$ Die meisten Menschen zogen aus dem näheren oder weiteren Umland nach Hamburg, ein nicht zu unterschätzender Teil kam jedoch von weiter her: aus England, den Niederlanden und Skandinavien, aus Frankreich, Italien und von der Iberischen Halbinsel. Die Fremden gaben der nach Wien zweitgrößten Stadt des Heiligen Römischen Reiches einen beinahe kosmopolitischen Charakter und trugen erheblich zu ihrer kulturellen Blüte im 17. und 18. Jahrhundert bei.

2 Georg Greflinger's Hamburgisches Reisehandbuch und Beschreibung von Hamburg im Gerist des Vereins für Hamburgische Geschichte 9 (1894), 122-149, hier 129

3 Vgl, etwa Stats- und Universitätsbibliothek Bremen, Mikrofilmsammlung der Zeitungen und zeitungsähnlichen Periodika, Wochentliche Zeitung aus mehrerley Orther (ohne Ort) 1630, Nr. 40; Reichs-Zeitungen (Hamburg?) 1630, Nr. 4; Post. Hamburger und Reichs-Zeitung (Hamburg) 1645, Nr. 50; Wochentliche Zeitung (ohne Ort), 1648, Nr. 10 u. 61 (Mikrofilme ja 2846 u. ja 3028/1) sowie Karl Heinrich Oberacker Jr.: Presse-Nachrichten über Brasilien aus dem 17. Jahrhundert. In: Staden-Jahrbuch 13 (1965), 141-144.

4 Julia Zunckel: Rüstungsgeschäfte im Dreißigjährigen Krieg. Unternehmerkräfte, Militärgïter und Marktstrategien im Handel zwischen Genua, Amsterdam und Hamburg. Berlin 1997.

5 Hans Mauersberg: Wirtschafts- und Sozialgeschichte zentraleuropäischer Städte in neuere Zeit, dargestellt an den Beispielen von Basel, Frankfurt a.M., Hamburg, Hannover und München. Göttingen 1960, 47 .
Während die wirtschaftlichen Beiträge der fremden Kaufleute in vielen Darstellungen Erwähnung finden und zum Teil umfassend erforscht sind, ${ }^{6}$ wurden die Handelsverbindungen der eingesessenen Hamburger Kaufmannschaft von der Frühneuzeit-Forschung in den letzten Jahrzehnten stark vernachlässigt. Zu Beginn des 20. Jahrhunderts war dies anders. Damals entstand eine Reihe wichtiger Arbeiten zur Hamburger Handelsgeschichte. Nach der 1871 erfolgten Reichsgründung bemühte sich die Geschichtsschreibung um die Erforschung der historischen Wurzeln des Nationalstaates. Hamburg wurde auch im Rückblick als Tor Deutschlands zur Welt betrachtet. Die Historiker deuteten die mittelalterlichen und frühneuzeitlichen Handelsbeziehungen als Vorläufer der zeitgenössischen imperialistisch-expansiven Ambitionen des Reichs. Der Überzeugung folgend, dass Deutschland zur Weltmacht berufen sei, wurde Hamburg sowohl in ökonomisch-kommerzieller als auch in flotten- und kolonialpolitischer Hinsicht eine Schlüsselrolle zugemessen. Noch zur Zeit der Weimarer Republik, des Nationalsozialismus und auch in der frühen Bundesrepublik erfolgte die Erforschung der Hamburger Handelsgeschichte aus einer ähnlichen Perspektive. Als Beispiele hierfür seien der 1925 veröffentlichte Aufsatz von Rudolf Häpke, Reichswirt schaftspolitik und Hanse, die 1929 erschienene Monographie von Ludwig Beutin, Hanse und Reich im handelspolitischen Endkampf gegen England, und das im selben Jahr von Erwin Wiskemann vorgelegte Buch, Hamburg und die Welthandelspolitik, genannt. ${ }^{7}$ Speziell mit Kaufleuten beschäftigte sich danach vor allem Percy Ernst Schramm. ${ }^{8}$ Für ihn war die hamburgische Geschichte eine groß angelegte Kaufmannsgeschichte. Als traditionsbewusster und stolzer Hamburge stellte er die Hamburger Kaufmannschaft unter dem Vorzeichen von „Leistung und „Beitrag“ dar. Er beschränkte sich dabei weitgehend auf seine eigene Familiengeschichte, die er grundsätzlich für exemplarisch hielt, womit er jedoch nur einen außerordentlich niedrigen Abstraktionsgrad erreichte. Es nimmt daher kaum Wunder, dass keines der genannten Werke von der internationalen Geschichtsschreibung rezipiert wurde. Dies gilt selbst für die 1975 veröffentlichte

6 Vgl. Wolf-Rüdiger Baumann: The merchants adventurers and the continental cloth-trade $(1560$ s-1620s). Florenz 1982; Robert van Roosbroeck: Die Niederlassung von Flamen und Wallonen in Hamburg (1567-1605). In: Zeitschrift des Vereins für Hamburgische Geschichte 49/50 (1964), 53-76; Hermann Kellenbenz: Unternehmerkräfte im Hamburger Spanienund Portugalhandel, 1590-1625. Hamburg 1954; ders.: Sephardim an der unteren Elbe. Thre wirtschaftliche und politische Bedeutung vom Ende des 16. bis zum Beginn des 18. Jahrhunderts. Wiesbaden 1958.

7 Rudolf Häpke: Reichswirtschaftspolitik und Hanse nach den Wiener Reichsakten des 16. Jahrhunderts. In: Hansische Geschichtsblätter 50 (1925), 164-209; Ludwig Beutin: Hanse und Reich im handelspolitischen Endkampf gegen England. Berlin 1929; Erwin Wiskemann: Hamburg und die Welthandelspolitik von den Anfängen bis zur Gegenwart. Hamburg 1929.

8 Percy Ernst Schramm: Neun Generationen. Dreihundert Jahre deutscher „Kulturgeschichte “ im Lichte der Schicksale einer Hamburger Bürgerfamilie (1648-1948). Göttingen 1963 f.; ders.: Gewinn und Verlust. Die Geschichte der Hamburger Senatorenfamilien Jancquel und Luis (16. bis 19. Jahrhundert). Zwei Beispiele für den wirtschaftlichen und sozialen Wandel in Norddeutschland. Hamburg 1969. Vgl 7u P. E. Schramm auch David Thimme: Die Erin. In: Zeitschrift des Vereins für Hamburgische Geschichte 89 (2003), 227-262. 
fachlich weit überzeugendere Studie von Martin Reißmann, Die hamburgische Kaufmannschaft des 17. Jahrhunderts in sozialgeschichtlicher Sicht.${ }^{9}$ Reißmann präsentiert darin zwar eine enorme Fülle wichtiger Daten, die Rolle der Hamburger Kaufleute als internationale Akteure analysiert er jedoch nicht.

Abgesehen von den Autoren einiger Einzeldarstellungen zum 18. und frühen 19. Jahrhundert ${ }^{10}$ war Hermann Kellenbenz der einzige Nachkriegshistoriker, der ausländische Archive in seine Forschungen einbezog und auf entsprechend breiter Basis rezipiert wurde. Sein international wohl am häufigsten zitiertes Werk sind die Sephardim an der unteren Elbe, das 1958 erschien, aber bereits 1944 verfasst worden war. ${ }^{11}$ Auch wenn Kellenbenz mit dem Buch keineswegs beabsichtigte, eine Geschichte der Hamburger Kaufmannschaft des 17. Jahrhunderts zu schreiben, nimmt es aufgrund des bestehenden Forschungsdefizites teilweise eine solche Funktion ein. Vor allem in der internationalen Geschichtsschreibung, in der fast ausschließlich dieses Buch als Referenzwerk zur hamburgischen Handelsgeschichte verwendet wird, wird den portugiesischen Juden daher oft eine im Verhältnis zu den anderen im Fernhandel tätigen Hamburger Akteuren zu große Bedeutung beigemessen. ${ }^{12}$ Erst in jüngster Zeit entstanden einige Arbeiten, die die Geschichte der eingesessenen Hamburger Kaufleute einschließlich ihrer internationalen Beziehungen in den Blick nehmen, wie z. B. die 2004 veröffentlichte Monographie von Klaus Weber, Deutsche Kaufleute im Atlantikhandel 16801830, und die 2007 erschienene Arbeit von Margrit Schulte Beerbühl, Deutsche Kaufleute in London 1660-1818. ${ }^{13}$ Beide Autoren betrachten die hamburgischen Kaufleute jedoch nach wie vor als „deutsche“ Kaufleute.

9 Martin Reißmann: Die hamburgische Kaufmannschaft des 17. Jahrhunderts in sozialgeschichtlicher Sicht. Hamburg 1975

10 U. a. Fred-Konrad Huhn: Die Handelsbeziehungen zwischen Frankreich und Hamburg im 18. Jahrhundert. Unter besonderer Berücksichtigung der Handelsverträge von 1716 und 1769. Hamburg 1953 (unveröff. Diss.); Hans Pohl: Die Beziehungen Hamburgs zu Spanie und dem spanischen Amerika in der Zeit von 1740 bis 1806 . Wiesbaden 1963; Rainer Ramcke: Die Beziehungen zwischen Hamburg und Osterreich im 18 reichsstädtisches Verhältnis im Zeichen von Handels- und Finanzinteressen. Hamburg 1969; Heinrich Ernst Köppen: Die Handelsbeziehungen Hamburgs zu den Vereinigten Staaten von Nordamerika bis zur Mitte des 19. Jahrhunderts. Köln 1973; Frauke Röhlk: Schiffahrt und Handel zwischen Hamburg und den Niederlanden in der zweiten Hälfte des 18. und zu Beginn des 19. Jahrhunderts. Wiesbaden 1973; Otto-Ernst Krawehl: Hamburgs Schiffs- un Warenverkehr mit England und den englischen Kolonien, 1814-1860. Köln 1977; Pierre Jeannin: Die Hansestädte im europäischen Handel des 18. Jahrhunderts. In: Hansische Geschichtsbïtter 89 (1971), 41-73; Ḧ̈ndler, Pioniere, amerika. Hrsg. von Jörn Arfs/Ulrich Mücke. Berlin 2010.

11 Kellenbenz, Sephardim (Anm. 6). Zur Entstehungsgeschichte vgl. Helmut Heiber: Walter Frank und sein Reichsinstitut für Geschichte des neuen Deutschlands. Stuttgart 1966, $456 \mathrm{f}$

Vgl. dazu ausführlicher meine demnächst erscheinende Dissertation mit dem Arbeitstitel: Kaufleute zwischen Hamburg und Portugal. Zur Bedeutung von Nation und Religion für das Leben und den Handel hamburgischer, niederländischer und portugiesischer Kaufleute im 17. Jahrhundert.

13 Klaus Weber: Deutsche Kaufleute im Atlantikhandel 1680-1830. Unternehmen und Familien in Hamburg, Cádiz und Bordeaux. München 2004; Margrit Schulte Beerbühl: Deutsche Kaufleute in London. Welthandel und Einbürgerung (1660-1818). München 2007.
Ich vertrete demgegenüber die Auffassung, dass es sich bei Hamburgs frühneuzeitlicher Handels- und Kaufmannsgeschichte um ein spezifisch hamburgisches Forschungsfeld handelt, das in Abgrenzung zur Geschichte der Kaufleute anderer Regionen zu untersuchen ist. Denn Hamburg betrieb in der Frühen Neuzeit eine relativ unabhängige Wirtschafts- und Außenpolitik, und weder das Reich noch die Hanse verfügten nach dem 16. Jahrhundert über Strukturen, die ihnen eine entsprechende Politik erlaubt hätten. Hamburg konnte bereits sehr früh die ,deutschen' Konsulate im Ausland mehr oder weniger eigenständig besetzen. ${ }^{14}$ Das älteste Konsulat, das dem deutschen Sprachraum zugeordnet werden kann, ist das 1571 erstmalig erwähnte hansische Konsulat in Lissabon. Bei den Verhandlungen, die im Vorfeld des Vertrags von 1607 zwischen der Hanse und dem spanisch-portugiesischen Reich stattfanden, sprach sich Hamburg mit aller Vehemenz gegen die Einrichtung eines traditionellen Hansekontors in Lissabon aus. ${ }^{15}$ So konnte die Stadt das Konsulat als dauerhafte Institution etablieren, die von der Hanse besetzt und finanziert werden sollte. Doch die Hanse verlor immer mehr an Bedeutung. Ab 1637 erfolgte die Entlohnung des Konsuls nicht mehr aus ihrer Gemeinschaftskasse, sondern anteilig durch die am Portugalhandel beteiligten Hansestädte. Hamburg steuerte die Hälfte des Geldes bei, da es das mi Abstand größte Handelsvolumen hatte. Entsprechend gelang es der Stadt bei der Wahl des Konsuls in Lissabon und anderen Hafenstädten immer leichter, ihre Interessen gegenüber den beiden verbleibenden Hansestädten Lübeck und Bremen durchzusetzen.

Auch in der Selbst- und Fremdwahrnehmung bildeten die Hamburger Kaufleute eine eigenständige, in sich geschlossene Gruppe, die entsprechend dem frühneuzeitlichen Sprachgebrauch zum Teil als ,Nation' bezeichnet wurde. Dies spiegelt sich beispielsweise in den Konversionsakten der portugiesischen Inquisitionsbehörde wider. ${ }^{16}$ Zwischen 1641 und 1691 wurden vom Lissabonner Inquisitionstribunal über 650 Konversionen von Ausländern aus protestantischen Gegenden registriert. Neben anderen Informationen zur Person musste jeder Konvertit seinen Herkunftsort sowie seine Nationszugehörigkeit angeben. Mit einem Anteil von $19 \%$ der in diesem Zeitraum aktenkundig gewordenen Konversionen bildeten die Hamburger nach den Engländern die zweitgrößte Gruppe. An dritter Stelle folgten mit einem Anteil von $10 \%$ die Angehörigen der ,deutschen Nation. Dabei wurden als zur , hamburgischen' Nation gehörig ausschließlich die tatsächlich aus Hamburg stammenden Personen gerechnet. Lübecker und Bremer

14 Georg Fink: Diplomatische Vertretungen der Hanse seit dem 17. Jahrhundert bis zur Auflösung der Hanseatischen Gesellschaft in Berlin 1920. In: Hansische Geschichtsblätter 56 (1931), 112-155; Ludwig Beutin: Zur Entstehung des deutschen Konsulatswesens im 16. und 17. Jahrhundert. In: Vierteljahrschrift für Sozial- und Wirtschaftsgeschichte 21 (1928) 438-448; Otto Beneke: Zur Geschichte des hamburgischen Consulatwesens. Hamburg 1866.

15 Archiv der Hansestadt Lübeck, ASA Externa, Hispanica 9.

16 Arquivo Nacional da Torre do Tombo (ANTT), Inquisição de Lisboa, Reduzidos, liv. 708715. Für eine detaillierte Ausführung vgl. meine demnächst erscheinende Dissertation (vgl. Anm. 12). 
wurden dagegen ebenso wie Frankfurter, Augsburger und Nürnberger, Westfalen, Brandenburger und Schlesier als Angehörige der deutschen Nation registriert.

Dass die Hamburger nicht als Deutsche angesehen wurden, zeigt auch ein Aussage des in Evora im Landesinneren von Portugal tätigen Konsuls der Deutschen und Flamen João Canjuel. Er denunzierte 1627 eine Gruppe Hamburger Kaufleute vor der Inquisition, weil sie lutherische Versammlungen abgehalten hätten, und erklärte dabei, dass sie sich fälschlicherweise als Deutsche ausgegeben hätten, in Wirklichkeit aber Hamburger seien und dass Hamburg nicht in Deutschland (Alemanha), sondern in „Germania Inferior ${ }^{66}$ liege. ${ }^{17}$

Meine Forschungen beschränken sich bislang auf die zwischen Hamburg und Portugal tätigen Kaufleute. Es finden sich jedoch auch immer wieder Hinweise auf die starke Präsenz von Hamburger Kaufleuten an vielen anderen Handelsstützpunkten, die in der Frühen Neuzeit von Bedeutung waren. Beispielsweise existieren für die Wende vom 16. zum 17. Jahrhundert Zeugnisse über die Anwesenheit von Hamburger Kaufleuten im brasilianischen Olinda. ${ }^{18}$ Von Olinda mit seinem Hafen Recife und von Salvador de Bahia aus wurde um diese Zeit fast der gesamte amerikanische Zucker nach Europa verschifft. Für die Jahre 1595 bis 1605 sind die Ausfuhrregister der in Recife abgehenden Schiffe erhalten. Mehr als die Hälfte von ihnen kamen aus Hamburg und wurden von Hamburger Schiffern gesteuert. Obwohl sie theoretisch dazu verpflichtet waren, über Portugal zurückzukehren, gab es immer wieder Schiffer, die auf dem direkten Weg nach Hamburg fuhren. Dort wurde die Zuckerverarbeitung schnell zu einem der führenden Gewerbezweige, was die Nachfrage nach Rohzucker immer weiter ansteigen ließ. Aus diesem Grund waren die Hamburger Kaufleute seit der zweiten Hälfte des 17. Jahrhunderts auch in der Karibik stark vertreten. Eine herausragende Rolle müssen sie um 1700 unter anderem auf der kleinen niederländischen Insel Saba gespielt haben, hielt doch ein englischer Schiffsschreiber die Insel irrtümlich für hamburgisch und notierte „Saba is the Hamburghers"“. ${ }^{19}$

Auch an der afrikanischen Küste waren Hamburger im 17. Jahrhundert tätig Ihr intensiver Schmuggel veranlasste 1651 den in Hamburg ansässigen Agenten der portugiesischen Krone, Duarte Nunes da Costa, den portugiesischen Überseerat dazu aufzufordern, über den Handel der Hamburger in den Häfen der von den Portugiesen reklamierten Besitzungen an der Küste Afrikas, Guineas und von Mina zu beraten. Da man davon ausging, den Handel der Fremden nicht grundsätzlich unterbinden zu können, wurde lediglich darüber debattiert, wie er am besten zu kontrollieren und mit angemessenen Abgaben zu belegen wäre. ${ }^{20}$

17 Canjuel selbst war gebürtiger Antwerpener (ANTT, Inquisição de Lisboa, liv. 212, 19-28v)

18 José Antônio Gonsalves Mello: Os Livros das Saídas das Urcas do Porto do Recife, 15951605. In: Revista do Instituto Arqueológico Histórico e Geográfico Pernambucano 58 (1993), 21-143.

19 Zit. n. Ernst Baasch: Beiträge zur Geschichte der Handelsbeziehungen zwischen Hamburg und Amerika. Hamburg 1892 (Hamburgische Festschrift zur Erinnerung an die Entdeckung Amerika's 1), 20.

20 Eulália Maria Lahmeyer Lobo: Aspectos da influência dos homens de negócio na política comercial ibero-americana, século XVII. Rio de Janeiro 1963 (unveröff. Diss.), $74 \mathrm{f}$
Die Hamburger waren weit verstreut und gut vernetzt. Ob es sich bei ihnen um eine Kaufmannsdiaspora handelte, ist angesichts der schwierigen Abgrenzung zu den Deutschen und Niederländern fraglich. Die Untersuchung solcher ethnischer Kaufmannsnetzwerke, von denen die der Araber, Armenier und Chinesen sowie die der von der Iberischen Halbinsel vertriebenen Neuchristen und Juden die bekanntesten sind, gehörte in den vergangenen 25 Jahren zu den wesentlichen Forschungsfeldern der globalen Sozial- und Wirtschaftsgeschichte. ${ }^{21}$ Die in diesen Netzwerken miteinander in Kontakt stehenden Personen waren durch eine gemeinsame Sprache, Kultur oder Religion und zum Teil auch durch eine geteilte historische Erfahrung der Vertreibung verbunden. In der Regel schlossen sie ihre Ehen innerhalb der eigenen Gruppe, sodass neben den ethnischen Gemeinsamkeiten oft auch Verwandtschaftsbeziehungen zwischen den Mitgliedern bestanden, die im Geschäftsleben eine wichtige Rolle für den Aufbau von Vertrauen spielten. Im Gegensatz zu den meisten anderen Handelsdiasporen stand mit der Stadt Hamburg jedoch eine Macht hinter den im Ausland lebenden Hamburgern, die sich gezielt für ihre Interessen einsetzte.

Auch die in Hamburg lebenden iberisch-jüdischen Kaufleute waren Teil einer Handelsdiaspora. Sie unterhielten u. a. Geschäftskontakte in ihre alte Heimat; im Gegensatz zu den hamburgischen Kaufleuten konnten sie aufgrund der Bedrohung durch die Inquisition nur unter großen Schwierigkeiten dorthin zurückkehren. Immer wieder wurden portugiesische Juden, die in Hamburg lebten, bei den iberischen Inquisitionstribunalen denunziert. Oft wurden dabei auch ihre Geschäftspartner auf der Iberischen Halbinsel angezeigt. Handelte es sich um Familienangehörige oder auch nur um Neuchristen, gerieten diese ebenfalls in die Gefahr der Verhaftung oder zumindest der Konfiszierung ihrer Güter. Die Kontakte in die ehemalige Heimat gehörten daher für die portugiesischen Juden zu den schwächsten beziehungsweise gefährdetsten Teilen ihrer Handelsnetzwerke. Aber auch in ihrer neuen Heimat hatten die Juden einen schwierigen Status, was unter anderem dadurch bedingt war, dass sich ihr Herkunftsstaat nicht für ihre Rechte einsetzte.

Hamburgs Fremdenpolitik in der Frühen Neuzeit wird gewöhnlich als tolerant bezeichnet. In der Forschung wurde bislang davon ausgegangen, dass andersgläubige Fremde, solange sie ihre Religion nicht in der Öffentlichkeit ausübten oder Unruhe in der Bevölkerung stifteten, mit den Hamburgern gleichberechtigt Handel treiben durften. ${ }^{22}$ Dass dem keineswegs so war, zeige ich in meiner in Kürze erscheinenden Dissertation. ${ }^{23}$ Durch das Gästehandelsverbot waren die Fremden gegenüber den Hamburger Bürgern erheblich benachteiligt. Dieses Verbot betraf insbesondere den Handel mit Getreide und Wein, den vom Ge-

21 Vgl. etwa die Überblickswerke Diaspora Entrepreneurial Networks. Four Centuries of History. Hrsg. von Ina Baghdiantz McCabe/Gelina Harlaftis/Ioanna Pepelasis Minoglou. Oxford 2005; Annales. Histoire, Sciences Sociales 58 (2003); Merchant networks in the early modern world Hrsg von Sanjay Subrahmanyam. Aldershot 1996; Philip Curtin: Cross-Cultural Trade in World History. Cambridge 1984

22 Vgl. etwa Jutta Braden: Hamburger Judenpolitik im Zeitalter lutherischer Orthodoxie 15901710. Hamburg 2001, 111

23 Vgl. Anm. 12. 
samthandelswert her bedeutendsten Waren, die zwischen Hamburg und der Tberischen Halbinsel in der ersten Hälfte des 17. Jahrhunderts ausgetauscht wurden. Ein Gast oder Fremder durfte diese Waren nicht von einem anderen Fremden kaufen, beziehungsweise an einen anderen Fremden verkaufen, sondern war dazu verpflichtet, das Geschäft mit einem Hamburger Bürger abzuwickeln. Die in den Fremdenkontrakten stehenden Portugiesen und Niederländer waren also nicht gänzlich vom Getreide- und Weinhandel ausgeschlossen. Wie eine statistische Auswertung der Hamburger Admiralitätszollbücher zeigt, waren sie jedoch bei den für den Gästehandel verbotenen Gütern weit unterrepräsentiert. Sie wichen stattdessen auf Waren aus, die 1604 für den Handel zwischen Gästen freigegeben worden waren, und handelten mit Wachs und Tuchen, Farbstoffen und Zucker.

Die in den Fremdenkontrakten stehenden Kaufleute waren zudem von den alten Fahrergesellschaften ausgeschlossen, in deren Rahmen sich die Hamburger Kaufleute trafen, sozialisierten und sicherlich auch Geschäftskontakte pflegten. Sie waren aber auch bezüglich der 1665 gegründeten Commerzdeputation erheblich benachteiligt, einer kaufmännischen ,Lobbygruppe', die von der Versammlung des sog. Ehrbaren Kaufmanns gewählt wurde. Während den nichtchristlichen Kaufleuten, insbesondere den portugiesischen Einwanderern jüdischen Glaubens, aber auch den Angehörigen des Englischen Court, der Zutritt zur Versammlung des Ehrbaren Kaufmanns verwehrt war, waren die im Fremdenkontrakt stehenden Niederländer zugelassen, selbst wenn sie dem reformierten Bekenntnis anhingen. ${ }^{24}$

Doch nicht nur die Commerzdeputation trat als Interessenvertretung der christlichen Hamburger Kaufmannschaft auf. Bei den auswärtigen Beziehungen übernahm diese Funktion vor allem der Rat der Stadt. Dessen Mitglieder waren zum Teil selbst Kaufleute, auf jeden Fall gehörten sie der städtischen Oligarchie an, die sich aus den wichtigsten Kaufmannsfamilien zusammensetzte. Die Außenpolitik des Rates wurde noch nicht systematisch untersucht, es ist jedoch davon auszugehen, dass sie sich in starkem Maße an den Interessen der Kaufmannschaft orientierte. Nachweislich unterhielten viele einflussreiche Personen aus der Hamburger Verwaltung und Regierung umfangreiche Handelsnetzwerke, andere hatten nahe Verwandte mit wichtigen wirtschaftlichen Positionen im Ausland. Dies zeigen unter anderem die folgenden Beispiele.

Johann Schröttering, 1588 in Hamburg geboren, war sowohl im Iberienhandel als auch in der Hamburger Politik stark engagiert. 1616 wurde er von der Hamburger Bürgerschaft zum Deputierten des Bakenzolls und acht Jahre später des Admiralitätszolls ernannt. 1627 wurde er Bankbürger, 1629 Kämmereibürger und 1638 Ratsherr. Nachdem im Wahlrezess von 1663 festgelegt worden war, dass „Unter den Herren Bürgermeister [...] einer ein Kaufmann sein [soll], welcher des Kauf- und See-Handels wohl kundig und erfahren“, ${ }^{25}$ wurde er 1667 als erster Kaufmann entsprechend dieser Vorgabe zum Bürgermeister gewählt. Wie

24 Ernst Baasch: Die Handelskammer zu Hamburg 1665-1915. Hamburg 1915, Bd. 1, 218, 544.

25 Johann Gustav Gallois: Hamburgische Chronik von den ältesten Zeiten bis auf die Jetztzeit. Bd. 3 (1618-1712). Hamburg 1870, 311 aus den Admiralitätszollakten der 1630er und 1640er Jahre ersichtlich wird, gehörte er zugleich zu den umsatzstärksten Iberienkaufleuten. ${ }^{26}$

Ein Jahr nach Schröttering wurde Johann Schulte zu einem weiteren der insgesamt vier Bürgermeister ernannt. ${ }^{27}$ Er selbst war zwar Jurist, doch unter seinen Söhnen und Schwiegersöhnen gab es mehrere bedeutende Kaufleute. Der bekannteste von ihnen, der den gleichen Namen wie sein Vater trug, wurde $1680 \mathrm{im}$ Alter von 18 Jahren für einige Zeit von seinen Eltern nach Lissabon geschickt, um dort seine in Hamburg begonnene kaufmännische Ausbildung abzuschließen, die portugiesische Sprache zu erlernen, Geschäftskontakte zu knüpfen und die Grundlage für sein späteres Handelsleben zu legen. Die Briefe des Vaters an den in Lissabon lebenden Sohn sind erhalten und geben ein eindrucksvolles Bild vom kaufmännischen Leben eines Hamburgers in der Fremde, aber auch von den Bemühungen des Bürgermeisters, von Hamburg aus die Geschäftsbeziehungen für den Sohn zu knüpfen beziehungsweise zu pflegen. ${ }^{28}$ Nach seiner Rückkehr aus Lissabon wurde Johann Schulte 1692 zum Commerzdeputierten ernannt. Auch einer seiner Söhne ließ sich später wieder in Lissabon nieder, ein jüngerer Bruder Johann Schultes war Konsul in Livorno, ein Schwager hatte in seiner Jugend ebenfalls als Kaufmann in Lissabon gelebt und war später von Hamburg aus sein Förderer gewesen.

Eine andere weit verzweigte, wirtschaftlich und politisch prominente Familie ist die aus den Niederlanden stammende Familie Heusch. Pieter Heusch war vor 1596 aus Antwerpen nach Hamburg eingewandert und gehörte in den 1630er Jahren zu den umsatzstärksten Portugalkaufleuten. ${ }^{29}$ Seine Söhne Jochem, Pieter und Michiel taten sich ebenfalls im Hamburger Portugalhandel hervor. Ein weiterer Sohn, Willem, wurde 1641, im Anschluss an die Erklärung der portugiesischen Unabhängigkeit von Spanien, vom neuen portugiesischen König zum deutschen Konsul in Lissabon ernannt. ${ }^{30}$ Da Portugal im Kampf gegen Spanien dringend Kredit und Waffen benötigte und diese über Hamburg leicht zu beziehen waren, war mit dem Posten des Konsuls zu dieser Zeit ein außergewöhnlich großer Einfluss verbunden. Das hohe Ansehen, das Willem Heusch am portugiesischen Hof genoss, wird unter anderem daran deutlich, dass er den späteren portugiesischen Staatsrat Gaspar de Faria Severim als Taufpaten für seinen Sohn Alexan-

$26 \mathrm{Zu}$ den wichtigsten Produkten seines Warenspektrums gehörte der Farbstoff Sumach, der zeitweise fast ein Fünftel seines gesamten Handelsvolumens im Iberienhandel ausmachte, zen Admertung der Admiralitatszollegister Zgl. meine in Küze erscheinde Diss. Anm. 12). In seiner Jugend hatte Schröttering „weite Reisen gemacht“, wie Friedrich Georg Buek notiert $\mathrm{Ob}$ diese ihn auch auf die Iberische Halbinsel führten, ist unbekannt. Vgl. Friedrich Georg Buek: Genealogische und biographische Notizen über die seit der Reformation verstorbenen hamburgischen Bürgermeister. Mit Facsimiles der Unterschriften. Hamburg 1840, 99-103.

27 Buek, Bürgermeister (Anm. 26), 103-111.

28 Briefe des Hamburgischen Bürgermeisters Johann Schulte Lt. an seinen in Lissabon etablierten Sohn Johann Schulte, geschrieben in den Jahren 1680-1685. Hrsg. von Ernst Merck. Hamburg 1856.

29 Vgl. die Auswertung der Admiralitätszollakten in meiner Dissertation (vgl. Anm. 12).

30 ANTT, Chancelaria de D. João IV, Doações, liv. 13, f. 5v; Tribunal do Santo Ofício (TSO), Concelho Geral (CG), Habilitações, Teodósio, mç. 1, doc. 10. 
der gewinnen konnte. ${ }^{31}$ Nach Willem Heuschs Tod trat Alexander 1669 dessen Nachfolge als Konsul an und blieb bis zu seinem eigenen Ableben im Jahr 1726 im Amt. In Hamburg hatte unterdessen der Bruder Willems, Michiel Heusch, zusammen mit einigen anderen Kaufleuten die Commerzdeputation gegründet. Wahrscheinlich stand er ihr von 1665 bis 1667 als Wortführer vor. ${ }^{32}$ Auch Michiel Heusch verfügte über ein außerordentlich großes Handelsnetzwerk. 1647 zahlte er Admiralitätszoll für Waren, die er aus 13 verschiedenen Häfen bezog bzw. dorthin versandte: Lissabon, Porto, Sanlúcar, Cádiz, Málaga, San Sebastián, Rouen, Marseille, Genua, Venedig, Sizilien, London und Danzig. Dies sind fast alle Häfen, für die zu diesem Zeitpunkt überhaupt Admiralitätszoll entrichtet werden musste.

Stellvertretend für viele weitere Beispiele von Hamburger Kaufleuten, die in erster oder zweiter Generation in die portugiesische Herrschaftselite aufstiegen, sei schließlich noch Peter Hasse genannt. Um 1620 in Hamburg geboren, kam er als etwa 16-Jähriger nach Lissabon, wo er zunächst von einem deutschen Kaufmann aufgenommen wurde. ${ }^{33}$ Später machte er sich als Geschäftsmann selbstständig und übernahm eine Reihe von Kronkontrakten, was ihn als Angehörigen der portugiesischen Wirtschaftselite ausweist. Zusammen mit seinem in Lissabon geborenen Sohn André war er maßgeblich an der Ausrüstung der Flotte beteiligt, die zur Rückeroberung Nordostbrasiliens von den Holländern eingesetzt wurde. Die Hasses gaben dem König dafür Kredit und besorgten Waffen und andere Gegenstände aus ,dem Norden', mit Sicherheit auch über Hamburg. Für seine Verdienste wurde André mit den höchsten Auszeichnungen bedacht, die ein Kaufmann in Portugal erlangen konnte: 1671 wurde er zum Christusritter und 1675 zum Familiar der Inquisition ernannt, schließlich 1691 in den Adelsstand erhoben. Außerdem wurde Hasse das Amt des Schatzmeisters der Junta do Comércio übertragen, einer Commerzdeputation für den Brasilienhandel, die jedoch unter staatlicher Leitung stand..$^{34}$

Wie gezeigt werden konnte, spielten Hamburger Kaufleute in der frühneuzeitlichen Weltwirtschaft eine bedeutende Rolle. Die Position Hamburgs unterschied sich grundlegend von der der Kolonialmächte Portugal, Spanien, England, Frankreich und der Niederlande. Seit Beginn des 17. Jahrhunderts unterschied sie sich auch von derjenigen der anderen Hansestädte oder italienischen Stadtstaaten, die den internationalen Handel bis zum 16. Jahrhundert mitbestimmt hatten. Die

31 Zum Zeitpunkt der Taufe war Gaspar de Faria Severim noch Secretário das Mercês, erst unter Afonso VI. wurde der einflussreiche Politiker und Neffe des berühmten Dichters Manuel Severim de Faria zum Staatsrat ernannt.

32 Baasch, Handelskammer (Anm. 24), 552.

33 ANTT, Registo Geral das Mercês, D. Pedro II, liv. 7, 80v; D. Pedro II, liv. 10, 293; D. João V, liv. 7, 571; ANTT, Habilitações da Ordem de Christo, letra A, mç. 45, doc. 72; TSO, CG, Habilitações, Andre, mç. 3, doc. 57; Teodósio, mç. 1, doc. 10; João, mç 11, doc. 338; Pedro, mç 12, doc. 299; Inquisição de Lisboa, Reduzidos, liv. 713, 307.

34 Wie die Beziehungen, die die Hasses nach Hamburg unterhielten, im Detail aussahen, ist nicht bekannt. Es bestand jedoch ein ständiger personeller Austausch zwischen Hamburg und Lissabon. Hamburger schickten ihre Söhne zur Ausbildung nach Lissabon, und die Söhne der in Lissabon niedergelassenen Deutschen reisten nach Hamburg. André Hasse hatte ne der in Lissabon niedergelassenen Deutschen reisten nach Hamburg. And
beispielsweise 1682 einen 15-jährigen Pagen aus Hamburg in seinem Dienst.
Diaspora der Hamburger Kaufleute weist zudem nur eine begrenzte Ähnlichkeit zu den ethnisch bestimmten Kaufmannsdiasporen auf. Die Rolle Hamburgs als einer weitgehend unabhängig operierenden Stadt ist in der frühmodernen Weltwirtschaft relativ einmalig. Ein erster Schritt zu ihrer Erforschung im globalen Kontext wäre die systematische Erfassung der Hamburgensien in den Archiven der europäischen und außereuropäischen Hafenstädte. Danach müssten länderübergreifende Untersuchungen unter thematischen Schwerpunkten durchgeführt werden. Denn Hamburgs Geschichte der Neuzeit, gleich ob wirtschaftlich, politisch, sozial oder kulturell, baut direkt oder indirekt auf der Stellung der Stadt im Weltzusammenhang auf. ${ }^{35}$

Eine globale Verortung Hamburgs ist auch notwendig, um den Anschluss an die internationale Geschichtsschreibung wiederzufinden, die sich in zunehmendem Maße von der nationalstaatlichen und eurozentrischen Sichtweise distanziert. Die Kolonial- und Imperialmächte werden heute längst nicht mehr als die einzigen gestaltenden Akteure gesehen. Vielmehr hat sich gezeigt, dass eigenverantwortlich handelnde, gut vernetzte und institutionell geförderte Kaufleute den Prozess der Globalisierung maßgeblich mitbestimmten. Unabhängig von ihrer individuellen Herkunft waren sie am weltweiten Aufbau der modernen Gesellschafts-, Wirtschafts- und Herrschaftsverhältnisse beteiligt. Ohne die finanziellen Erträge aus dem Überseehandel und die kulturellen Erfahrungen, die die Kaufleute aus der ganzen Welt mit nach Hause brachten, hätte sich Hamburg nicht zu dem entwickeln können, was es heute ist. Hamburgs Geschichte ist nur zu verstehen vor dem Hintergrund seiner auswärtigen Kontakte, Interaktionen und globalen Verflechtungen. Denn Hamburger kamen bereits in der Frühen Neuzeit bis ,in die äusserste Welt Oerther"

35 Sichtbar wurde dies u. a. auf der Konferenz „Portugal, Hamburg und die deutschsprachige Welt während der europäischen Expansion nach Übersee“, die im Juni 2009 vom Instituto Camões an der Universität Hamburg in Zusammenarbeit mit dem Centro de História de Além-Mar der Universidade Nova de Lisboa ausgerichtet wurde. 